\title{
Complicaciones quirúrgicas del abordaje endoscópico endonasal transesfenoidal en macroadenomas hipofisarios no funcionantes: estudio de centro único
}

\author{
Surgical complications of the transsphenoidal endonasal endoscopic approach to \\ pituitary macroadenomas: single center study
}

\author{
César A. Almendárez-Sánchez ${ }^{*}$, Hugo García-Velasco', Miguel A. Ramírez-Sosa1, \\ Carlos A. Tevera-Ovando', Javier López-Zapata², Milton Ruiz-Flores' y Raúl Huato-Reyes ${ }^{1}$ \\ 'Departamento de Neurocirugía, Centro Médico "Lic. Adolfo López Mateos", Instituto de Salud del Estado de México, Toluca, Estado de México; \\ 2Instituto Nacional de Neurología y Neurocirugía "Manuel Velasco Suárez", Ciudad de México. México
}

\begin{abstract}
Resumen
Antecedentes: Los abordajes quirúrgicos de la región selar han sido un reto para los neurocirujanos. Con la introducción de la técnica endoscópica se ha minimizado el trauma quirúrgico, pero esta técnica no se encuentra exenta de complicaciones. Objetivo: Describir nuestra experiencia institucional en pacientes sometidos a resección de macroadenomas hipofisarios por vía microquirúrgica endonasal transesfenoidal asistida por endoscopia con énfasis en las complicaciones resultantes. Método: Revisamos los expedientes electrónicos de 17 pacientes sometidos a dicho procedimiento de manera consecutiva entre 2017 y 2018, y recabamos variables como edad, sexo, clasificación imagenológica, tiempo de diagnóstico al momento de la cirugía, complicaciones posoperatorias y recurrencia. Resultados: La edad promedio de los pacientes fue de 45.23 años y el $70.59 \%$ eran mujeres. Radiológicamente predominó el grado 2 Knosp (47.05\%). La complicación principal fue diabetes insípida transitoria (23.52\%), seguida por la fístula de líquido cefalorraquídeo (11.76\%). Conclusiones: El manejo microquirúrgico asistido por endoscopia ha demostrado ser un recurso valioso para tratar macroadenomas hipofisarios, pero presenta grados variables de complicaciones, por lo cual se requiere la adquisición de habilidades para el uso del endoscopio, la planeación preoperatoria adecuada y el manejo conjunto multidisciplinario con el fin de evitarlas.
\end{abstract}

Palabras clave: Endoscopia. Adenoma hipofisario. Complicaciones quirúrgicas.

\begin{abstract}
Background: Surgical approaches to the sellar region have been a challenge for the neurosurgeon. With the introduction of the endoscopic technique, surgical trauma has been minimized, however it is not free of complications. Objective: To describe our institutional experience in patients undergoing resection of pituitary macroadenomas by endoscopic-assisted microsurgical approach, with emphasis on the resulting complications. Method: We reviewed the electronic files of 17 patients who underwent this procedure consecutively between 2017 and 2018, collecting variables such as age, sex, imaging classification, time of diagnosis at the time of surgery, postoperative complications and recurrence. Results: The average age of the patients was
\end{abstract}

\section{Correspondencia:}

*César A. Almendárez-Sánchez

Avda. Nicolás San Juan s/n

Ex Hacienda La Magdalena

Fecha de recepción: 05-06-2020

C.P. 50010, Toluca, Edo. de México, México

E-mail: cesar2hx@hotmail.com

0009-7411/@ 2020 Academia Mexicana de Cirugía. Publicado por Permanyer. Este es un artículo open access bajo la licencia CC BY-NC-ND (http://creativecommons.org/licenses/by-nc-nd/4.0/). 
45.23 years, with $70.59 \%$ women. Radiologically, grade 2 Knosp predominated (47.05\%). The main complication was transient diabetes insipidus (23.52\%) followed by cerebrospinal fluid fistula (11.76\%). Conclusions: The endoscopic-assisted microsurgical approach has proven to be a valuable resource for treating pituitary macroadenomas, however it presents variable degrees of complications, which requires the acquisition of skills for the use of the endoscope, adequate preoperative planning, and multidisciplinary joint management in order to avoid them.

Key words: Endoscopy. Pituitary adenoma. Surgical complications.

\section{Introducción}

Los abordajes quirúrgicos de la región selar y paraselar han representado un desafío para los neurocirujanos por muchos años. La introducción del abordaje endoscópico es un reflejo de la evolución de la neurocirugía, en busca de evitar craneotomías amplias utilizando abordajes cada vez mas pequeños y por ende reducir las complicaciones asociadas al trauma quirúrgico y con ello obtener mejores resultados posoperatorios.

La primera resección de una lesión de la región selar fue descrita por Schloffer en 1907'. Con el advenimiento del microscopio quirúrgico, Hardy describió en 1962 la técnica microquirúrgica transesfenoidal para la resección de dichas lesiones ${ }^{2}$, la cual fue el manejo utilizado con mayor frecuencia durante la segunda mitad del siglo pasado, hasta la introducción de la técnica endoscópica endonasal transesfenoidal descrita por Jankowski en $1992^{3}$. En la actualidad, dicho abordaje es ampliamente utilizado en todo el mundo, pero no está exento de complicaciones.

En el presente trabajo presentamos la experiencia institucional en pacientes sometidos a resección de macroadenomas hipofisarios por vía microquirúrgica endonasal transesfenoidal asistida por endoscopia durante 2 años consecutivos (2017 y 2018), con énfasis en las complicaciones inmediatas y mediatas asociadas a dicho abordaje.

\section{Método}

Realizamos una cohorte retrospectiva, mediante la revisión de los expedientes electrónicos de todos los pacientes sometidos a resección de adenomas hipofisarios por vía microquirúrgica endonasal transesfenoidal asistida por endoscopia en nuestra institución, el Centro Médico Licenciado Adolfo López Mateos, de manera consecutiva desde enero de 2017 hasta diciembre de 2018. Incluimos solo pacientes con expediente clínico completo, cuyas lesiones contaron con reporte histopatológico confirmatorio de adenoma hipofisario, excluyendo otros tipos de lesiones, con un tamaño mayor de $10 \mathrm{~mm}$ (macroadenomas) y con valoración endocrinológica previa que clasificara la lesión como no funcionante, siendo 17 pacientes los que cumplían con estos criterios de inclusión.

Las variables recabadas de los pacientes fueron la edad, el sexo, la clasificación imagenológica (Hardy-Vezina y Knosp), el tiempo de diagnóstico al momento de la cirugía y las complicaciones posoperatorias en las primeras 2 semanas, así como el seguimiento a 6 meses.

El análisis estadístico fue realizado por dos investigadores independientes. Toda la información fue colectada en una hoja de cálculo de Microsoft Excel (Office 2016, Microsoft) y después analizada con IBM SPSS (Version 21, IBM).

\section{Resultados}

\section{Características preoperatorias de los pacientes}

Fueron 17 pacientes los incluidos en este estudio (Tabla 1 ), de los cuales 12 eran mujeres $(70.59 \%)$ y 5 eran hombres (29.41\%). La edad promedio de todos los pacientes fue de 45.23 años: 46.3 años paras las mujeres (rango: 28-62) y 42.6 años para los hombres (rango: 22-55). El promedio de tiempo con diagnóstico de macroadenoma hipofisario previo a la cirugía fue de 6.9 meses. El 23.52\% (5 casos) presentaban hipotiroidismo concomitante previo a la realización de la cirugía.

En cuanto a la clasificación Knosp (Tabla 2), utilizada para valorar el grado de invasión al seno cavernoso, identificamos que el $47.05 \%$ (8) de los pacientes presentan un grado 2, seguido por el grado 4 en el $23.52 \%$ (4) y en último término los grados 3 y 2 (17.64\% y $11.76 \%$, respectivamente).

Con relación a la clasificación Hardy-Vezina modificada (Tabla 2), en cuanto al grado de invasión local a la silla turca observamos que el $52.94 \%$ (9) de los 
Tabla 1. Características preoperatorias de los pacientes con macroadenomas hipofisarios incluidos en el estudio

\begin{tabular}{lc}
\hline Características $\mathbf{N}=\mathbf{1 7}$ & \\
\hline Sexo & \\
Femenino, $\mathrm{n}(\%)$ & $12(70.59)$ \\
Masculino, $\mathrm{n}(\%)$ & $5(29.41)$ \\
& \\
Edad promedio (años) & \\
Femenino & $46.3(28-62)$ \\
Masculino & $42.6(22-55)$ \\
\hline
\end{tabular}

Tabla 2. Relación del número de macroadenomas hipofisarios intervenidos en el periodo de estudio con respecto a la clasificación imagenológica otorgada

\begin{tabular}{lcc}
\hline \multicolumn{3}{c}{ Clasificación Knosp } \\
\hline 1 & $\mathbf{n}$ & $\mathbf{( \% )}$ \\
\hline 2 & 2 & 11.76 \\
3 & 8 & 47.05 \\
4 & 3 & 17.64 \\
\hline Silla turca & Clasificación Hardy-Vezina modificada \\
\hline II & $\mathbf{n}$ & $\mathbf{( \% )}$ \\
III & 3 & 17.64 \\
IV & 9 & 52.94 \\
\hline Extensión extraselar & 5 & 29.41 \\
\hline B & $\mathbf{n}$ & $\mathbf{( \% )}$ \\
C & 4 & 23.52 \\
D & 7 & 41.17 \\
E & 5 & 29.41 \\
\hline
\end{tabular}
pacientes presentaban grado III, el $29.41 \%$ (5) grado
IV y el $17.64 \%$ (3) grado II. Respecto a la extensión
extraselar, el $41.17 \%$ (7) fueron catalogados dentro
de la clasificación como grupo C, el grupo D vino a
continuación en orden de frecuencia con el $29.41 \%$
(5), posteriormente el grupo B con el $23.52 \%$ (4) y en
último lugar el grupo E con solo un caso (5.88\%).

\section{Complicaciones inmediatas y mediatas}

Identificamos en total siete diferentes complicaciones en nuestros pacientes (Fig. 1). En cuanto a las

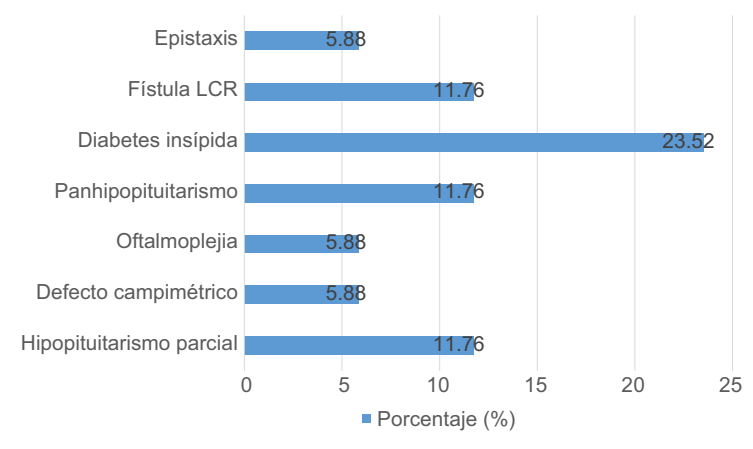

Figura 1. Comparación de las complicaciones posoperatorias en los pacientes sometidos a resección de macroadenoma hipofisario no funcionante por vía microquirúrgica endonasal transesfenoidal asistida por endoscopia.

que se presentaron desde el momento de la cirugía hasta 2 semanas posteriores, es decir, complicaciones inmediatas y mediatas, la más frecuente fue la diabetes insípida en el $23.52 \%$ (4), con requerimiento de vasopresina. La misma fue transitoria y remitió en el seguimiento médico posterior en todos los pacientes. Tras ella encontramos la fístula de líquido cefalorraquídeo (LCR) en el $11.76 \%$ (2) de los casos. En estos pacientes se colocó un catéter lumbar subaracnoideo por 3 a 5 días y se indicó reposo absoluto, con remisión de la misma posteriormente en ambos casos. El panhipopituitarismo y el hipopituitarismo parcial se presentaron igualmente, en el $11.76 \%$ (2) de los casos, con requerimiento de terapia hormonal sustitutiva. Como complicaciones menos frecuente encontramos epistaxis (resuelta adecuadamente con taponamiento nasal), oftalmoplejía y defectos campimétricos, con un caso cada una (5.88\%); los dos últimos sin mejoría en las valoraciones subsecuentes.

\section{Recidiva tumoral}

En 3 (17.64\%) pacientes se logró documentar el crecimiento de la lesión en los siguientes 6 a 12 meses, por lo cual fueron sometidos de nuevo a manejo quirúrgico endoscópico transesfenoidal, sin complicaciones posoperatorias reportadas.

\section{Discusión}

El acceso a la región selar tiene limitantes anatómicas y quirúrgicas importantes, pues se encuentra por encima el quiasma óptico y el polígono de Willis, lateralmente está rodeada por el seno cavernoso y 
Tabla 3. Incidencia de complicaciones asociadas a la resección de macroadenomas hipofisarios por vía endoscópica endonasal con relación a las reportadas en la literatura mundial

\begin{tabular}{|c|c|c|c|c|c|c|c|c|c|}
\hline \multicolumn{10}{|c|}{ N. ${ }^{\circ} \operatorname{casos}(\%)$} \\
\hline \multirow[t]{2}{*}{ Autores } & \multirow[t]{2}{*}{ Fístula LCR } & \multicolumn{3}{|c|}{ Diabetes insípida } & \multirow{2}{*}{ Epistaxis } & \multirow{2}{*}{$\begin{array}{l}\text { Disfunción } \\
\text { hipofisaria }\end{array}$} & \multirow{2}{*}{$\begin{array}{l}\text { Perforación } \\
\text { tabique nasal }\end{array}$} & \multirow{2}{*}{ Meningitis } & \multirow[t]{2}{*}{ Muerte } \\
\hline & & Total & Temporal & Permanente & & & & & \\
\hline Shen, et al. ${ }^{12}$ & 0 & $2(5)$ & $2(5)$ & 0 & 0 & 0 & NA & 0 & 0 \\
\hline Jho, et al..$^{13}$ & $10(8)$ & $11(9)$ & $6(5)$ & $5(4)$ & 0 & $18(14)$ & NA & 0 & 1 \\
\hline Cappabianca, et al. ${ }^{14}$ & $3(2)$ & $13(9)$ & $8(5)$ & $5(3)$ & $2(1)$ & $20(14)$ & NA & 0 & 0 \\
\hline White, et al..$^{15}$ & $6(12)$ & $11(22)$ & $9(18)$ & $2(4)$ & $1(2)$ & 0 & 0 & $1(2)$ & 0 \\
\hline Rudnik, et al. ${ }^{11}$ & 0 & $3(5)$ & NA & $3(5)$ & 0 & NA & NA & 0 & 1 \\
\hline Caster, et al. ${ }^{10}$ & $4(27)$ & $3(20)$ & $3(20)$ & 0 & $1(7)$ & 0 & $3(20)$ & 0 & 0 \\
\hline Kabil, et al. ${ }^{16}$ & $5(2)$ & $16(5)$ & $12(4)$ & $4(1)$ & $4(1)$ & $8(3)$ & $2(1)$ & 0 & 0 \\
\hline Serie actual & $2(11.76)$ & $4(23.52)$ & $4(23.52)$ & 0 & $1(5.88)$ & $2(11.76)$ & 0 & 0 & 0 \\
\hline
\end{tabular}

LCR: líquido cefalorraquídeo; NA: no aplica.

por detrás hay estructuras vitales, como la arteria basilar y sus respectivas ramas ${ }^{4}$. Tradicionalmente se había utilizado la técnica microquirúrgica para el abordaje de esta región, pero desde hace dos décadas el uso de la cirugía endoscópica endonasal transesfenoidal para lesiones pituitarias ha sido uno de los avances más remarcables, dado que representa una disminución en el número de complicaciones y evita procedimientos con morbilidad alta, como las craneotomías ${ }^{5}$. Entre sus ventajas se encuentran la adecuada visualización de la anatomía, un campo quirúrgico amplio y la facilidad para modificar el ángulo de trabajo con el endoscopio.

Los adenomas son la lesión más común de la región selar y representan un $10-15 \%$ de todas las lesiones intracraneales, lo cual implica un porcentaje alto de patología neuroquirúrgica ${ }^{6}$. Los factores anatómicos de la pared lateral del seno cavernoso desempeñan un papel importante en la invasión tumoral, por lo que la resonancia magnética es de vital importancia para determinar el comportamiento de la lesión a resecar. Teniendo en cuenta esta premisa, utilizamos la clasificación de Knosp, con la cual encontramos que el $47.05 \%$ de nuestros pacientes fueron clasificados como grado 2, extendiéndose por fuera de la línea intercarotídea, sin sobrepasar la tangente de la carótida intrasupracavernosa.

Otro factor importante es identificar el grado de invasión a la silla turca, lo cual se logra con la clasificación de Hardy-Vezina modificada, con la que evidenciamos que el $52.94 \%$ de los pacientes presentó perforación focalizada del piso selar (grado III). En cuanto a la extensión extraselar, la variante mas frecuente fue el grupo C, la cual se refiere a un desplazamiento importante del piso del tercer ventrículo, con el $41.17 \%$ de los casos.

\section{Complicaciones endocrinológicas}

Son sin duda las complicaciones más frecuentes reportadas en todo el mundo, siendo la diabetes insípida la más predominante. Esta se ha atribuido a la disfunción de neuronas productoras de vasopresina por la manipulación quirúrgica. Dicha alteración amerita revisión y seguimiento endocrinológico ulterior, aunque suele tener una recuperación completa en los 6 meses posteriores, tal como ocurrió en nuestros $4(23.52 \%)$ casos. Otra complicación en este rubro es el hipopituitarismo parcial (adenohipófisis) o completo (panhipopituitarismo), el cual suele ocurrir por un uso excesivo de la aspiración o bien por coagulación de la cavidad selar con daño al tejido glandular sano peritumoral.

\section{Complicaciones infecciosas}

La meningitis es una complicación infrecuente, reportada en el $0-2 \%$ de los $\operatorname{casos}^{8}$. La meningitis va de la mano con la fístula de LCR posquirúrgica, y su presencia es el principal factor de riesgo dado que es la vía de entrada de microorganismos patógenos al 
sistema nervioso central. Ningún paciente de nuestra serie presentó dicha complicación.

\section{Complicaciones estructurales}

Durante el abordaje, el piso de la silla turca es eliminado o drilado, con el fin de acceder a la duramadre y posteriormente al adenoma; sin embargo, esta maniobra puede generar una fístula de LCR. Encontramos en nuestra serie $2(11.76 \%)$ pacientes que presentaron dicha alteración, la cual fue manejada con éxito con un catéter subaracnoideo. El grado de esta complicación en nuestra institución se mantiene en los rangos reportados en la literatura mundial ${ }^{9}$. Suele tratarse de manera conservadora con un catéter lumbar subaracnoideo, y en caso de que no remita amerita reintervención quirúrgica.

\section{Complicaciones nasales}

La más frecuente reportada es la epistaxis, la cual suele deberse a una lesión de la arteria esfenopalatina, por lo cual una coagulación adecuada de esta es de suma importancia. Dicha complicación se ha reportado hasta en un $7 \%$ de los casos $^{10}$. Nosotros reportamos 1 (5.88\%) caso, atribuido a la manipulación quirúrgica durante la resección del macroadenoma, que se resolvió con taponamiento nasal.

Otras complicaciones reportadas en la literatura son anosmia e hiposmia hasta en el $10 \%$ de los pacientes $^{8}$; sin embargo, en nuestra serie no observamos tal alteración. Además, se ha documentado como complicación la perforación septal hasta en el 3\% de los pacientes ${ }^{8}$. En nuestra serie tampoco se produjo esta complicación.

\section{Complicaciones vasculares}

Una de las complicaciones más temidas por el neurocirujano al realizar este abordaje es la lesión de la arteria carótida interna. No obstante, esta complicación ha disminuido considerablemente tras la introducción de la neuroendoscopia y en la literatura mundial se reporta en menos del $1 \%$ de los casos ${ }^{11}$. Afortunadamente, en ninguno de nuestros pacientes se presentó dicha complicación.

De manera general, podemos comentar que el índice de complicaciones que encontramos en nuestro estudio es similar al reportado en las series internacionales (Tabla 3) $)^{11-16}$.

\section{Conclusión}

La mejor estrategia para tratar las complicaciones es evitarlas. Esto depende del adecuado entendimiento de la enfermedad, del conocimiento detallado de la anatomía quirúrgica de la región selar y paraselar, de la adquisición de habilidades para el manejo del endoscopio, de una adecuada planeación preoperatoria individualizando cada caso, del uso de instrumentos quirúrgicos adecuados, del seguimiento posquirúrgico regular y del trabajo coordinado multidisciplinario entre el neurocirujano, el endocrinólogo, el otorrinolaringólogo, el radiólogo, el patólogo y el radiooncólogo, y con ello conseguir el éxito en el manejo de nuestros pacientes. Es prácticamente imposible eliminar por completo las complicaciones debido a las estructuras circundantes en la región selar y paraselar, pero si se siguen esas pautas se pueden disminuir de manera considerable.

\section{Agradecimientos}

Los autores agradecen al personal médico involucrado en el manejo integral de los pacientes.

\section{Conflicto de intereses}

Los autores declaran no tener conflictos de intereses.

\section{Responsabilidades éticas}

Protección de personas y animales. Los autores declaran que para esta investigación no se han realizado experimentos en seres humanos ni en animales.

Confidencialidad de los datos. Los autores declaran que han seguido los protocolos de su centro de trabajo sobre la publicación de datos de pacientes.

Derecho a la privacidad y consentimiento informado. Los autores declaran que en este artículo no aparecen datos de pacientes.

\section{Bibliografía}

1. Tunkel A, Schloffer H. Erfolgreiche operation eines hypophysen tumors auf nasalem wege. Wien Klin Wochenschr. 1907;20:621-4. 
2. Linfoot JA. Transsphenoidal microsurgical treatment of pituitary tumours En: Recent advances in the diagnosis and treatment of pituitary tumour. New York: Raven Press; 1979. p. 375-888.

3. Jankowski R, Auque J, Simon C, Marchal JC, Hepner H, Wayoff M. Endoscopic pituitary tumor surgery. Laryngoscope. 1992;102:198ロ202.

4. Rhoton ALJ. The Sellar Region. Neurosurgery. 2002;51(S4):s1-335-74.

5. Tabaee A, Anand VK, Barrón Y, Hiltzik DH, Brown SM, Kacker A, et al. Endoscopic pituitary surgery: a systematic review and meta-analysis. J Neurosurg. 2009;111:545ロ54.

6. Cheng Y, Xue F, Wang TY, Ji JF, Chen W, Wang ZY, et al. Analyses and treatments of postoperative nasal complications after endonasa transsphenoidal resection of pituitary neoplasms. Medicine (Baltimore). 2017;96:e6614

7. Sudhakar N, Ray A, Vafidis JA. Complications after trans-sphenoidal surgery: our experience and a review of the literature. $\mathrm{Br} \mathrm{J}$ Neurosurg. 2004;18:507-12.

8. Charalampaki P, Ayyad A, Kockro RA, Perneczky A. Surgical complications after endoscopic transsphenoidal pituitary surgery. J Clin Neurosci. 2009;16:786-9.
9. Wang F, Zhou T, Wei S, Meng X, Zhang J, Hou Y, et al. Endoscopic endonasal transsphenoidal surgery of 1,166 pituitary adenomas. Surg Endosc. 2015;29:1270ø80.

10. Casler JD, Doolittle AM, Mair EA. Endoscopic surgery of the anterior skull base. Laryngoscope. 2005;115:16-24.

11. Rudnik A, Zawadzki T, Wojtacha M, Bazowski P, Gamrot J, Galuszka-Ignasiak B, et al. Endoscopic transnasal transsphenoidal treatment of pathology of the sellar region. Minim Invasive Neurosurg. 2005;48:101-7.

12. Shen CC, Wang YC, Hua WS, Chang CS, Sun MH. Endoscopic endonasal transsphenoidal surgery for pituitary tumors. Chin Med J Engl. 200;63:301-10.

13. Jho HD. Endoscopic transsphenoidal surgery. J Neurooncol. 2001;54(2):187-195.

14. Cappabianca P, Cavallo LM, Colao A, de Divitiis E. Surgical complications associated with the endoscopic endonasal transsphenoidal approach for pituitary adenomas. J Neurosurg. 2002;97(2):293ロ98.

15. White DR, Sonnenburg RE, Ewend MG, Senior BA: Safety of minimally invasive pituitary surgery (MIPS) compared with a traditional approach. Laryngoscope. 2004;114:1945-48.

16. Kabil MS, Eby JB, Shahinian HK. Fully endoscopic endonasal vs. transseptal transsphenoidal pituitary surgery. Minim Invasive Neurosurg. 2005;48:348-54. 